\title{
Distributed interference-aware admission control and resource allocation for underlaying D2D communications in cellular networks
}

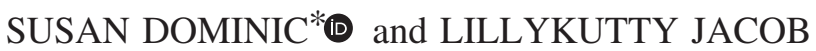 \\ Department of Electronics and Communication Engineering, National Institute of Technology Calicut, \\ Kozhikode 673601, India \\ e-mail: susandominic89@gmail.com; lilly@nitc.ac.in
}

MS received 6 August 2018; revised 7 February 2019; accepted 4 April 2019; published online 7 May 2019

\begin{abstract}
In this letter, we present a two-phase distributed resource allocation algorithm for a Device-toDevice (D2D) network underlaying a cellular network. In the first phase, D2D pairs learn resource block (RB) of the cellular user equipment (CUEs) to be used, while playing an interference minimization game. Each D2D pair is ensured an interference below an upper limit, which is chosen as the criterion for admission control. In the next phase, power allocation for the device pairs allocated to each RB is carried out to maximize their sum rate while ensuring a minimum rate to the CUEs using a pricing scheme. We perform simulations to evaluate the performance of the proposed scheme.
\end{abstract}

Keywords. Interference minimization; game theory; pricing.

\section{Introduction}

Device-to-Device (D2D) communication underlaying cellular networks is one of the promising methods for meeting the spectrum deficit and speed requirements of the future fifth generation (5G) networks. Devices communicate directly by reusing the resource blocks (RBs) allocated to cellular user equipment (CUEs). This leads to improving the spectral efficiency, capacity and energy efficiency while lowering the latency and spectrum usage. However, these benefits can be achieved only if the interference arising due to reuse is effectively mitigated through judicious resource allocation schemes.

Centralized resource allocation schemes for underlay D2D networks have been widely discussed in literature [1]. They require the Base Station (BS) to carry out the resource allocation for every device in the network. The computational and signalling overhead make these schemes inefficient in the future ultra-dense networks where pilot contamination is going to be a major hindering factor for the channel state information (CSI) acquisition. Distributed schemes requiring little-to-no network knowledge are hence desirable in the future.

In [2], authors propose a distributed resource allocation algorithm to guarantee a minimum data rate to the CUEs as well as D2D pairs. The admissible D2D pairs are required

*For correspondence to satisfy an interference condition, to have a feasible solution to the resource allocation problem, which may not be met. Also, the interference from the CUEs is ignored to determine the transmit power of the D2D pairs. Authors of [3] formulate the resource allocation problem as a Stackelberg game and obtain a decentralized solution that ensures a minimum rate to the CUEs and maximizes the sum throughput of the D2D pairs. The proposed scheme does not provide any quality of service (QoS) guarantees to the D2D pairs. In [4], a distributed resource allocation scheme is proposed to solve the same problem as in [3]. BS controls the interference by charging a reuse price to ensure a QoS for the CUEs. A non-cooperative power control game is proposed to allocate transmit powers to the D2D pairs. The proposed scheme requires information exchange among D2D pairs to determine best responses. A hybrid resource allocation scheme is proposed in [5], where the channels are allocated to the D2D pairs in a centralized manner by solving a graph matching problem. A Q-learning algorithm enables the D2D pairs to learn their transmit powers in a distributed manner while maximizing their sum rate. A power minimization game is also proposed to ensure a QoS for the D2D pairs in the form of a minimum rate guarantee. This, however, imposes a restriction on the interference among the D2D pairs. It also requires the interference from the $\mathrm{BS}$ to be negligibly small enough to be ignored.

In this paper, we propose an interference-aware admission control and a pricing-based power control for ensuring data rate above a minimum to the CUEs and interference below an upper limit to the D2D pairs. The proposed 
algorithm maximizes the sum rate of the D2D tier while meeting the QoS constraints of the CUEs and the D2D pairs. Different from those in the afore-mentioned literature, we introduce an interference limit as an admission criterion for the D2D pairs. This does not impose any restrictions on the topology of the network unlike [2] and [5]. The proposed algorithm ensures a minimum data rate to the CUEs and also a QoS guarantee to the D2D pairs unlike [3] and [4]. The contributions of this letter are the following:

1. A new admission control criterion for the D2D pairs that does not impose any restriction on the topology of the network.

2. A fully distributed Stochastic Learning Algorithm (SLA)-based [6] RB allocation scheme that ensures interference level below an upper limit and that does not require the $\mathrm{D} 2 \mathrm{D}$ pairs to have any information regarding the RB choices made by the other D2D pairs.

3. A pricing-based power control algorithm to allocate transmit powers to the D2D pairs while ensuring a minimum rate to the CUEs and maximizing the sum throughput of the D2D tier.

4. Performance comparison of the proposed scheme with the hybrid scheme in [5] and a centralized exhaustive search scheme.

\section{System model}

We consider a single cell with BS at the centre and CUEs and D2D pairs placed randomly in the cell. The BS is assumed to transmit to the CUEs at a constant power of $P_{o}$. Each CUE is allocated an RB for the BS to communicate to them in the downlink. The D2D pairs are assumed to reuse these downlink RBs of the CUEs. The CUEs and their respective $\mathrm{RBs}$ are indexed as $c \in\{1,2, \ldots, C\}$. The D2D pairs are indexed as $i \in\{1,2, \ldots, D\}$. The D2D pairs are permitted to transmit at any one of the $K$ discrete transmit powers $P_{1}<P_{2}<\cdots<P_{K}$. The channel gain between any two network entities (BS, CUE or D2D pair) $u$ and $v$ on an RB $c$ is $h_{u v}^{c}=g_{u v} f_{u v}^{c}$ where $0<g_{u v}<1$ is the path loss and $0<f_{u v}^{c}<1$ is the gain due to fading on RB $c$. The SINR of the CUE on $\mathrm{RB} c$ is

$$
\gamma^{c}=\frac{P_{o} h_{o c}^{c}}{\sum_{i=1}^{D} P_{i} h_{i c}^{c} \delta(c, i)+N_{0}}
$$

where the indicator function $\delta(c, i)$ takes value one if the D2D pair $i$ transmits on RB $c$ and zero otherwise. Similarly, the SINR of any D2D pair $i$ on $\mathrm{RB} c$ is

$$
\gamma_{i}^{c}=\frac{P_{i} h_{i i}^{c}}{\sum_{j \neq i} P_{j} h_{j i}^{c} \delta(c, j)+P_{o} h_{o i}^{c}+N_{0}}
$$

where $P_{o} h_{o i}^{c}$ is the interference from the BS.

\section{Interference-aware admission control and RB allocation}

In the first phase of the proposed two-phase resource allocation scheme, the D2D pairs learn the RBs to be used via SLA while minimizing the net interference of the D2D tier and ensuring an upper bound on the interference experienced by every D2D pair. A D2D pair $i$ decides to reuse $\mathrm{RB} c$ as long as $I_{i} \leq I^{\text {th }}$, where $I_{i}$ is as given in the denominator of (2). This is modelled as an interference minimization game $G_{I}=\left\{\mathscr{D},\left(\mathscr{L}_{i}\right)_{i \in \mathscr{D}},\left(u_{i}\right)_{i \in \mathscr{D}}\right\}$, where $\mathscr{D}$ is the set of D2D pairs; $\mathscr{L}_{i}$ is the set of actions available to D2D pair $i$, which is nothing but the set of $\mathrm{RBs} \mathscr{C}$; and $u_{i}$ is the utility achieved, which is the interference suffered by D2D pair $i$. Since the D2D pairs have no knowledge regarding the environment, they learn the $\mathrm{RB}$ allocation using SLA while transmitting at the maximum power of $P_{K}$. The utility of a D2D pair $i$ using RB $c$ is defined as

$$
u_{i}\left(l_{i}, \mathbf{l}_{-i}\right)=-I_{i}=-\left(\sum_{j \neq i} P_{K} h_{j i}^{c} \delta(c, j)+P_{o} h_{o i}^{c}\right)
$$

where $l_{i} \in \mathscr{L}_{i}$ is the action choice of D2D pair $i$ and $\mathbf{l}_{-i}$ is the joint action profile of all D2D pairs other than $i$. During the learning, each D2D pair $i$ maintains a probability vector over the action choices available (initialized with equal probabilities) and these probabilities are updated according to the instantaneous reward, $0 \leq r_{i} \leq 1$, it gets when the actions are chosen. The reward $r_{i}$ is determined as

$$
r_{i}=\max \left(0, \frac{\mathrm{I}_{\mathrm{th}}-\mathrm{Ii}}{\mathrm{I}_{\mathrm{th}}}\right)
$$

Smaller the interference, farther it is from $I_{t h}$ and higher the reward. At the end of the learning process, each D2D pair whose interference constraint is satisfied chooses an RB with probability one. The ones whose interference constraints are not satisfied receive zero rewards, and hence the initial probabilities remain unchanged. They decide to no longer participate in the resource allocation game and hence have to choose other modes such as overlay or cellular.

The interference minimization game can be proved to be an exact potential game with the potential function $\Phi\left(l_{i}, \mathbf{l}_{-i}\right)=-\frac{1}{2} \sum_{i} I_{i}$ by assuming the channel gains to be symmetric. The game is hence guaranteed to have at least one Nash Equilibrium (NE). The convergence of SLA can be proved as in [6].

\section{Pricing-based power allocation}

In the second phase, D2D pairs with allocated RBs have to learn their transmit powers, while satisfying the rate requirement of the respective CUEs, $R^{c} \geq R^{\text {th }}$. An iterative 
pricing-based power allocation algorithm is proposed for this. During each iteration of the price update algorithm, BS sets prices for the RBs and D2D pair $i$ on RB $c$ chooses a transmit power to maximize a function, $f_{i}\left(P_{i}\right)$, of the rate achieved and the price to be paid. Every D2D pair solves the optimization problem

$$
\max _{P_{i} \in\left\{P_{1}, P_{2}, \ldots, P_{K}\right\}} f_{i}\left(P_{i}\right)
$$

where

$$
f_{i}\left(P_{i}\right)=R_{i}^{c}\left(P_{i}\right)-\Theta_{c} P_{i} h_{i o}^{c}
$$

where $R_{i}^{c}$ is the rate obtained when the interference is $I_{t h}$. Such a choice of rate does not require the knowledge of the transmit power choices of the other D2D pairs on RB $c$. The second term represents the price paid and is proportional to the interference it generates at the CUE on the RB $c$. The prices are updated using a bisection algorithm as in [3]. The BS monitors the rates of the CUEs and updates the prices depending on whether $R^{c} \geq R_{t h}$ or not. The updated prices are broadcasted and the D2D pairs calculate their own transmit powers. The channel gain information $h_{i o}^{c}$ is assumed to be provided by the BS, once the RB allocation is completed.

Proposition 1 The proposed two-phase distributed resource allocation results in the maximization of the sum rate of the $\mathrm{D} 2 \mathrm{D}$ tier.

(i) In the first phase, each D2D pair minimizes the interference it suffers while transmitting at the maximum power. Hence each D2D pair maximizes its own average rate.

(ii) The sum of the lower bounds of the individual rates can be proved to be better than the lower bound of the sum rate of the D2D tier.

(iii) In the second phase, each D2D pair maximizes the function defined in (6), which is a function of only its own power. Hence the individual maximization is equivalent to maximizing the sum of such functions over all the D2D pairs.

Proof Proof is given in the Appendix.

\section{Simulation set-up and results}

We simulate the proposed algorithm for a cell of size $500 \mathrm{~m}$ $\times 500 \mathrm{~m}$. Rayleigh fading channels and normalized channel gains are considered. Other simulation parameters are given in table 1 . The performance of the proposed scheme is compared with the hybrid scheme in [5]. In figure 1, 'hybrid scheme (sum rate)' and 'hybrid scheme (QoS)' refer to two different objectives of power allocation: to maximize the sum rate of the $\mathrm{D} 2 \mathrm{D}$ tier and to ensure a minimum rate $R_{D}$ to each D2D pair $i$, respectively. Rate of the CUE is
Table 1. Simulation parameters.

\begin{tabular}{lc}
\hline Parameter & Value \\
\hline BS transmit power $P_{o}$ & $43 \mathrm{dBm}$ \\
D2D pair transmit power levels & $\{0,10,17,20,23\} \mathrm{dBm}$ \\
Path loss exponent & 3.3 \\
Noise power spectral density & $-174 \mathrm{dBm} / \mathrm{Hz}$ \\
$R_{t h}$ of CUEs & $3.11 \mathrm{E}+06 \mathrm{bps}$ \\
D2D pair tx-rx distance & $25 \mathrm{~m}$ \\
\hline
\end{tabular}

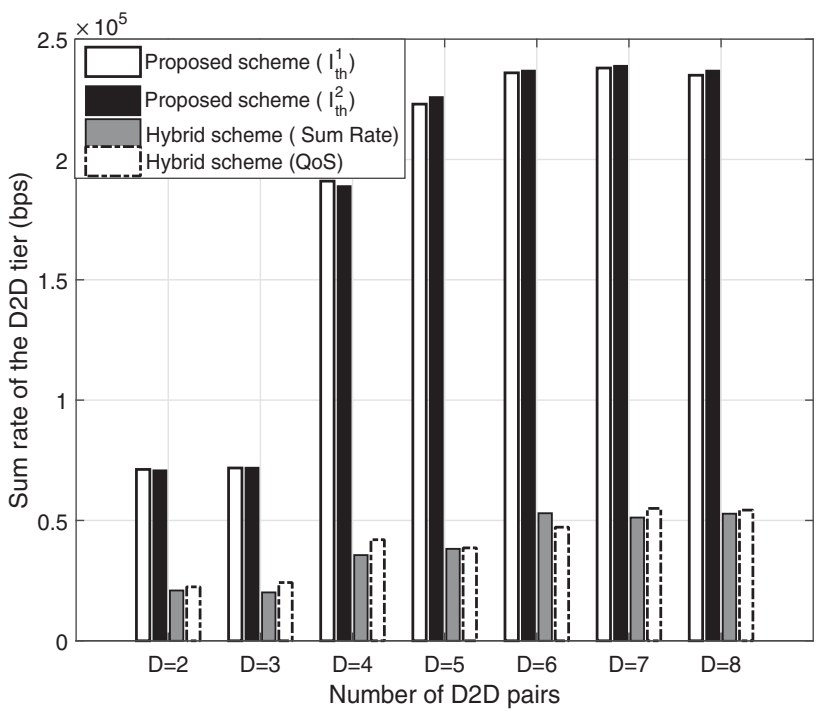

Figure 1. Sum rate of the D2D tier for different numbers of D2D pairs when $C=3$.

ensured to be above $R_{t h}$ in both cases. For the proposed scheme, we consider two interference thresholds, $I_{t h}^{1}$ and $I_{t h}^{2}$, corresponding to transmit power $P_{K}$ (maximum) and $P_{1}$ (minimum), respectively, and carry out the resource allocation as proposed.

As can be observed from figure 1, the proposed scheme preforms better than the hybrid scheme in terms of

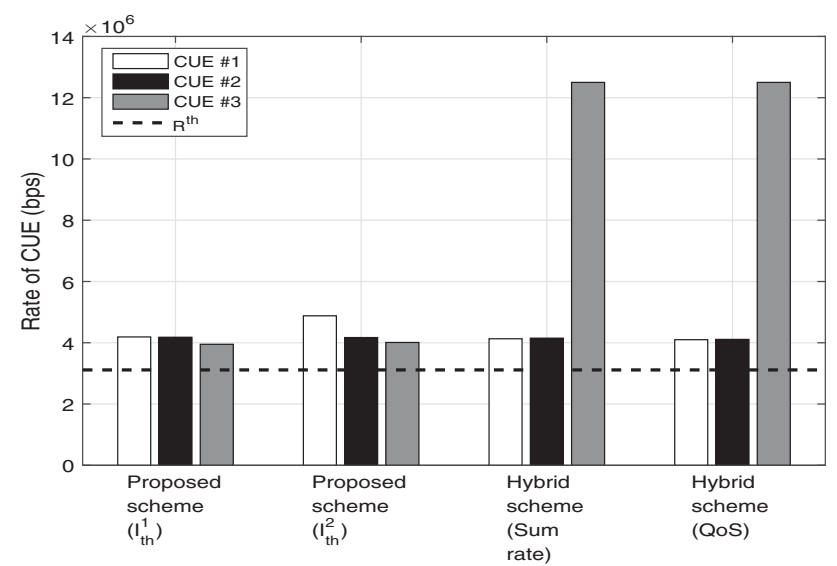

Figure 2. Rate of the CUEs when $D=8$ and $C=3$ for the various schemes. 
Table 2. Comparison of the sum rate of the D2D tier for the proposed scheme and centralized search scheme $(C=2)$.

\begin{tabular}{ccc}
\hline$D$ & Centralized exhaustive search & Proposed scheme \\
\hline 2 & $1.04 \mathrm{E}+05$ & $0.87 \mathrm{E}+05$ \\
3 & $1.04 \mathrm{E}+05$ & $0.88 \mathrm{E}+05$ \\
4 & $4.15 \mathrm{E}+05$ & $3.02 \mathrm{E}+05$ \\
5 & $4.25 \mathrm{E}+05$ & $3.12 \mathrm{E}+05$ \\
6 & $4.60 \mathrm{E}+05$ & $3.31 \mathrm{E}+05$ \\
\hline
\end{tabular}

the sum rate of the D2D tier while ensuring the interference threshold for the D2D pairs as well as the rate threshold of the CUEs. This can be observed in figure 2, where the rates of the 3 CUEs are compared with $R_{t h}$ for the various schemes. It can be seen that both the proposed scheme as well as the hybrid scheme satisfy the rate requirement of all the CUEs.

In table 2, we compare the proposed scheme with a centralized exhaustive search scheme for the same values of $R_{t h}$ and $I_{t h}$. As can be observed from the table, the proposed scheme gives at least $70 \%$ of the performance of the exhaustive search scheme, without the overhead of the information exchange among the D2D pairs.

\section{Conclusion}

We propose a distributed learning algorithm to pair the D2D pairs to the CUEs based on an interference threshold as an admission criterion. A pricing-based distributed power allocation is then carried out to ensure a minimum rate to all the CUEs and to maximize the sum rate of the D2D tier. We performed simulations to evaluate the performance of the proposed algorithm and found that it gives at least $70 \%$ of the sum rate obtained by the centralized exhaustive search scheme while satisfying the QoS of the CUE without any information exchange among D2D pairs.

\section{Appendix I.}

\section{Proof of Proposition 1}

(i) The average rate achieved by a $\mathrm{D} 2 \mathrm{D}$ pair $i$ on $\mathrm{RB}$ $c$ is

$$
R_{i}=B \log _{2}\left(1+\bar{\gamma}_{i}^{c}\right)
$$

where $\bar{\gamma}_{i}^{c}$ is the average SINR defined as follows:

$$
\bar{\gamma}_{i}^{c}=\frac{P_{i} \bar{h}_{i i}^{c}}{I_{i}+N_{0}}
$$

and $B$ is the bandwidth of the RB. The average gain $\bar{h}_{i i}^{c}$ is assumed to be equal over all RBs. When the D2D pair learns the RB index that ensures a minimum interference below the threshold of $I_{t h}$, while transmitting at the maximum power, we can say that it is maximizing the average SINR since the numerator of (A2) is maximized while its denominator is minimized.

(ii) From [7] we have the inequality

$$
\frac{x}{1+x} \leq \log _{2}(1+x) \leq x \forall x>-1 .
$$

Hence, we have

$$
\log _{2}\left(1+\bar{\gamma}_{i}^{c}\right) \geq \frac{\bar{\gamma}_{i}^{c}}{1+\bar{\gamma}_{i}^{c}}
$$

The lower bound in (A4) can be expressed as

$$
\frac{\bar{\gamma}_{i}^{c}}{1+\bar{\gamma}_{i}^{c}}=\frac{1}{1+\frac{1}{\bar{\gamma}_{i}^{c}}} \text {. }
$$

Hence, when each D2D pair maximizes the average SINR while learning the RB index (from (i)), it maximizes the lower bound in (A4).

Consider the sum rate of the D2D tier with just two D2D pairs $\left(\bar{\gamma}_{1}^{c}=x_{1}\right.$ and $\left.\bar{\gamma}_{2}^{c}=x_{2}\right)$.

$$
\begin{aligned}
\sum_{i \in \mathscr{D}} R_{i} & =B \log _{2}\left(1+x_{1}\right)+B \log _{2}\left(1+x_{2}\right) \\
& =B \log _{2}\left(\left(1+x_{1}\right)\left(1+x_{2}\right)\right) \\
& =B \log _{2}\left(1+\left(x_{1}+x_{2}+x_{1} x_{2}\right)\right) .
\end{aligned}
$$

From (A3)

$$
\log _{2}\left(1+\left(x_{1}+x_{2}+x_{1} x_{2}\right)\right) \geq \frac{x_{1}+x_{2}+x_{1} x_{2}}{1+x_{1}+x_{2}+x_{1} x_{2}} .
$$

On the other hand

$$
\frac{x_{1}}{1+x 1}+\frac{x_{2}}{1+x_{2}}=\frac{x_{1}+x_{2}+2 x_{1} x_{2}}{1+1+x_{1}+x_{2}+x_{1} x_{2}} \text {. }
$$

Clearly RHS of (A8) $>$ RHS of (A7), i.e., adding the lower bounds of individual rates gives a rate better than lower bound of the sum rate given by (A7). This is true for any number of D2D pairs.

Hence, in the first phase, instead of directly maximizing the sum rate, we maximize the rates achieved by individual D2D pairs and achieve a sum rate better than the lower bound of the sum rate. Since the upper bound in (A3) is nothing but the average SINR, we achieve the same upper bound in the proposed scheme. Hence, we can conclude that we achieve a better lower bound while maintaining the upper bound for the sum rate of the D2D tier.

(iii) In the second phase, each $\mathrm{D} 2 \mathrm{D}$ pair computes its rate by assuming the interference to be $I_{t h}$, i.e., the rate it can achieve in the worst case interference. Since the utility obtained by solving the optimization problem in (5) is 
dependent only on the transmit power of each D2D pair, maximization of individual utility results in the maximization of the sum utility. Hence the second phase ensures maximization of the sum rate of the D2D tier.

\section{References}

[1] Mach P, Becvar Z and Vanek T 2015 In-band device-todevice communication in OFDMA cellular networks: a survey and challenges. IEEE Commun. Surveys Tutor. 17: 1885-1922

[2] Yin R, Yu G, Zhang H, Zhang Z and Li G Y 2015 Pricing-based interference coordination for D2D communications in cellular networks. IEEE Trans. Wireless Commun. 14: 1519-1532
[3] Yin R, Zhong C, Yu G, Zhang Z, Wong K K and Chen X 2016 Joint spectrum and power allocation for D2D communications underlaying cellular networks. IEEE Trans. Veh. Technol. 65: 2182-2195

[4] Nguyen H, Hasegawa M and Hwang W 2016 Distributed resource allocation for D2D communications underlay cellular networks. IEEE Commun. Lett. 20: 942-945

[5] Maghsudi S and Stanczak S 2016 Hybrid centralizeddistributed resource allocation for device-to-device communication underlaying cellular networks. IEEE Trans. Veh. Technol. 65: 2481-2495

[6] Dominic S and Jacob L 2018 Distributed resource allocation for D2D communications underlaying cellular networks in time-varying environment. IEEE Commun. Lett. 22: 388-391

[7] Love E R 1980 Some logarithm inequalities. Math. Gazette 64: $55-57$ 